\title{
Efecto del pH, velocidad del aire y concentración de espumante sobre la cinética de flotación de cuatro carbones colombianos
}

\author{
Jorge L. Piñeres*\$, Juan M. Barraza* \\ Escuela de Ingeniería Química, Universidad del Valle, Cali, Colombia \\ §e-mail: jopime@hotmail.com
}

(Recibido: Junio 03 de 2008 - Aceptado: Noviembre 06 de 2008)

\begin{abstract}
Resumen
Este trabajo muestra el efecto del $\mathrm{pH}$, velocidad del aire y concentración de espumante sobre la constante cinética de flotación de cuatro muestras de carbones bituminosos colombianos: Cerrejón K60 (Guajira), Nechí M1 (Antioquia), La Jagua 15 (Cesar) y Guachinte (Valle del Cauca). Una columna de flotación de $5 \mathrm{~m}$ de altura se utilizó en todos los experimentos. Las muestras se procesaron usando un rango de $\mathrm{pH}$ entre 4 y 10 , una velocidad del aire entre 0.7 y $2.1 \mathrm{~cm} / \mathrm{s}$ y una concentración de espumante en el rango de 2 a $6 \mathrm{ml}$ de espumante / $\mathrm{kg}$ de carbón, respectivamente. Los carbones La Jagua y Cerrejón mostraron altos valores de la constante cinética en medio ácido. Sin embargo, el mayor valor obtenido para el carbón Cerrejón fue $0.43 \mathrm{~min}^{-1}$ a pH 7. Al contrario de lo ocurrido con los carbones La Jagua y Cerrejón, los carbones Nechí y Guachinte exhibieron altos valores de la constante cinética en medio básico. No obstante, al igual como ocurrió con el carbón Cerrejón, el mayor valor obtenido para el carbón Nechí fue $0.36 \mathrm{~min}^{-1}$ a pH 7 .
\end{abstract}

Palabras clave: Columna de flotación, Constante cinética, Concentración de espumante, Efecto del $\mathrm{pH}$.

CHEMICAL ENGINEERING

\section{Effect of pH, air velocity, and frother concentration on the flotation kinetics of four Colombian coals}

\begin{abstract}
This work shows the effect of $\mathrm{pH}$, air velocity and frother concentration on the flotation kinetic constant of four Colombian bituminous coal samples: Cerrejón K60 (Guajira), Nechí M1 (Antioquia), La Jagua 15 (Cesar) y Guachinte (Valle del Cauca). A flotation column of $5 \mathrm{~m}$ was used for all the runs. The samples were processed in a $\mathrm{pH}$ range of 4 to 10 , air velocity in the range of 0.7 to $2.1 \mathrm{~cm} / \mathrm{s}$ and frother concentration in the range of 2 to $6 \mathrm{ml}$ of frother $/ \mathrm{kg}$ of coal, respectively. The Jagua and Cerrejón coals showed high values of the kinetic constant using acid conditions. However, the largest value of the kinetic constant for Cerrejón coal $\left(0.43 \mathrm{~min}^{-1}\right)$ was reached at $\mathrm{pH} 7$. On the contrary, Nechí and Guachinte coals exhibited high values of the kinetic constant at basic conditions. Nonetheless, as it happened with Cerrejón coal, the largest value of the kinetic constant for Nechí coal $\left(0.36 \mathrm{~min}^{-1}\right)$ was reached at $\mathrm{pH} 7$.
\end{abstract}

Keywords: Flotation column, Kinetic constant, Frother concentration, Effect of pH. 


\section{Introducción}

Un desarrollo significativo en el proceso de flotación con el paso de los años es el incremento en el uso de las columnas de flotación en diversas industrias de minerales. Una columna de flotación se diferencia dramáticamente de una celda convencional en su diseño y filosofía de operación, siendo atractiva en aquellos procesos de limpieza donde se requieren múltiples etapas, debido a que una columna de flotación puede remplazar múltiples celdas de flotación, generando un circuito más simple y fácil de controlar.

Actualmente, las columnas de flotación son ampliamente utilizadas en los procesos de limpieza de carbón, basándose principalmente en las diferencias de las propiedades superficiales de sus componentes. Durante la flotación, las partículas con superficie hidrofóbica se adhieren a las burbujas de aire (materia orgánica) y son llevadas a la corriente de flotados (en la cima de la columna), en tanto que las partículas hidrofílicas (materia mineral) se obtienen en la corriente de descarga (en el fondo de la columna).

El carbón es un material extremadamente heterogéneo compuesto de materiales orgánicos e inorgánicos. A la fracción orgánica la representan los macerales, en tanto que a la fracción inorgánica la conforman la materia mineral y azufre. La flotabilidad de la fracción orgánica depende de varios factores tales como la composición petrográfica, grado de carbonización, oxidación de la superficie, entre otros (Leonard, 1991; Blaschke, 1996).

La evaluación de la constante cinética de flotación es de suma importancia para el cálculo de la longitud de una columna de flotación, así como para la determinación de sus parámetros de mezclado. En general, los principios de la cinética química son normalmente usados en el modelado del proceso de flotación, particularmente, la ecuación básica de velocidad representada por la siguiente expresión (Dobby \& Finch, 1986; Finch \& Dobby, 1990):

$$
\frac{d C}{d t}=-k C^{n}
$$

donde $C$, en el caso de una reacción química, representa la concentración del reactivo, $t$ es el tiempo, $k$ es la constante de velocidad y $n$ es el orden de la reacción. En el caso de la flotación de partículas hidrofóbicas, puede utilizarse una ecuación similar a la que se usa en reacciones químicas, cambiando $C$ por $m$ que es la masa de las partículas flotables remanentes en la celda. $\mathrm{La}$ similitud entre las ecuaciones para $C$ y $m$ se justifica por la suposición de que el volumen dentro de la columna de flotación permanece constante. Para columnas de flotación que operan con bajos flujos de aire y pequeños diámetros de burbuja el modelo de dispersión en flujo pistón hace una buena descripción del mezclado dentro de la columna. Sin embargo, el número de dispersión se calcula asumiendo que el coeficiente de dispersión axial de las partículas es equivalente al del líquido. Para condiciones de flujo pistón y cinética de primer orden, Dobby \& Finch (1986) y Finch \& Dobby (1990) presentan la siguiente ecuación:

$$
R_{f}=1-\exp \left(-k \tau_{p}\right)
$$

donde $\tau_{p}$ representa el tiempo de residencia del sólido, $k$ es la constante de velocidad y $R_{f}$ es la recuperación fraccional del material flotado. Yoon $\&$ Mao (1996) demostraron que $k$ es una función de los parámetros hidrodinámicos y superficiales que ocurren durante la flotación y que puede representarse como:

$$
k=\frac{3}{4}\left(\frac{J_{g}}{R_{b}}\right)\left[\frac{3}{2}+\frac{4 \mathrm{Re}_{b}^{0.72}}{15}\right]\left(\frac{R_{p}}{R_{b}}\right)^{2} \exp \left(-\frac{E_{p b}}{E_{k}}\right)
$$

donde $R_{p}$ y $R_{b}$ son los radios de la partícula y burbuja, respectivamente, $R e_{b}$ es el número de Reynolds de la burbuja, $E_{k}$ es la energía cinética de la partícula, $J_{g}$ es la velocidad superficial del aire y $E_{p b}$ es la energía de barrera entre la partícula y la burbuja. El objetivo de este trabajo fue evaluar experimentalmente las constantes cinéticas de flotación de cuatro muestras de carbón: Cerrejón, La Jagua, Nechí y Guachinte, usando una columna de flotación a escala piloto, con la finalidad de diseñar y construir columnas de flotación para limpieza de carbones colombianos. 


\section{Metodología}

\subsection{Materiales}

Cuatro muestras de carbones colombianos se utilizaron en este estudio: Cerrejón K60 (Guajira), Nechí M1 (Antioquia), La Jagua 15 (Cesar) y Guachinte (Valle del Cauca). Las muestras (tal como fueron recibidas) se sometieron a un proceso de molienda hasta obtener un tamaño de partícula menor a malla $400(38 \mu \mathrm{m})$. Aceite de creosota, lauril éter sulfato de sodio al $28 \%$, hidróxido de sodio $(\mathrm{NaOH} 1.7 \mathrm{M})$ y acido clorhídrico $(\mathrm{HCl} 3.3$ M) se utilizaron como colector, espumante y modificadores del $\mathrm{pH}$, respectivamente.

\subsection{Equipo}

La columna de flotación utilizada se fabricó en acrílico y tiene $5 \mathrm{~m}$ de altura y $0.05 \mathrm{~m}$ de diámetro. La entrada del flujo de alimentación se ubicó a una altura de $1.2 \mathrm{~m}$ medida desde la cima de la columna. El sistema de generación de burbujas, que consta de un burbujeador poroso fabricado en vidrio, se colocó a una altura de 0.38 m medida desde el fondo de la columna. La entrada del flujo de aire se ubicó a esta misma altura, en tanto que la entrada del flujo de espumante se ubicó a $0.66 \mathrm{~m}$ del fondo de la columna. La recolección del material flotado se realizó por medio de un vertedero ubicado a una distancia de $0.22 \mathrm{~m}$ de la cima, el cual tiene un diámetro de $0.25 \mathrm{~m}$ y una altura de $0.40 \mathrm{~m}$ de frente y $0.20 \mathrm{~m}$ por detrás de la columna, con el objeto de facilitar la evacuación del mineral. En la Figura 1 se muestra un esquema del equipo utilizado.

\subsection{Experimentación}

Una vez la mezcla agua-carbón se homogeniza por recirculación, se abre la válvula de alimentación hasta obtener el flujo deseado. Cuando la mezcla alcanza $0.23 \mathrm{~m}$ (aproximadamente) por encima de la alimentación, se abre la válvula del rotámetro de aire asegurándose que el manómetro indique 14.7 psi y el flujo de aire sea el deseado. Luego se ajustan los flujos de espumante y agua, para mantener la altura deseada por encima de la alimentación. El tiempo total de la operación es de 20 min (aproximadamente) desde el momento en

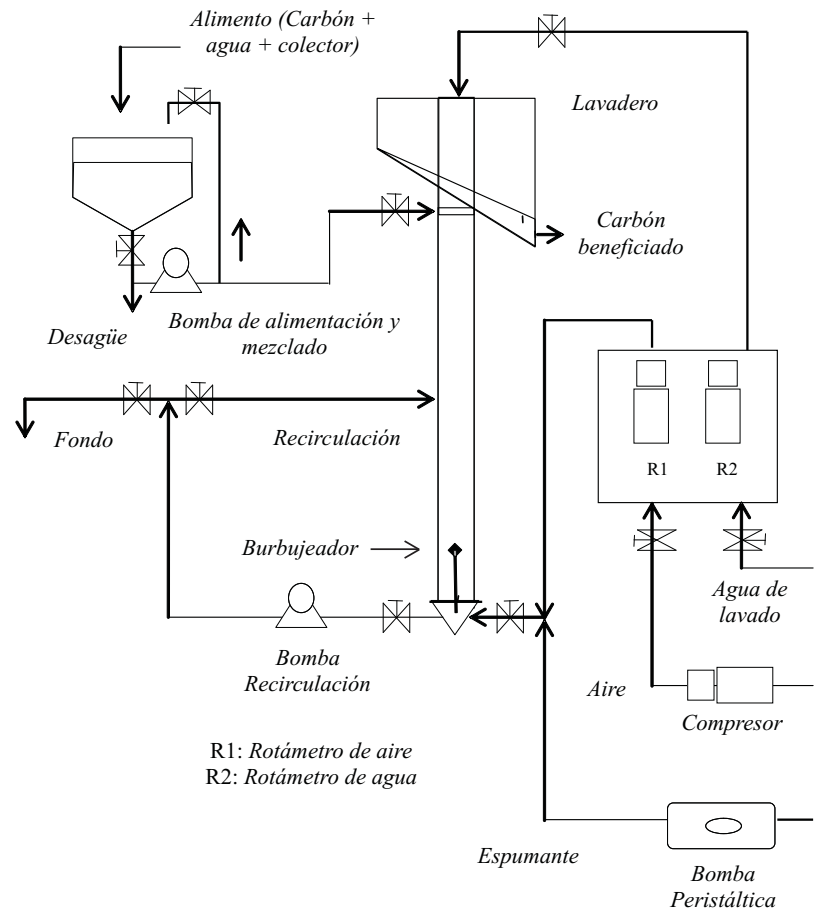

Figura 1. Esquema de la columna de flotación utilizada.

que la primera cantidad de carbón llega a la zona de recolección de la cima de la columna. Las corrientes de cima y de fondo se recogen en recipientes y posteriormente se filtran.

Las muestras de carbón se secaron y almacenaron para realizarles los análisis necesarios. Durante las corridas se tomaron muestras a intervalos de $4 \mathrm{~min}$. Todas las corridas se realizaron usando una sola etapa, con una concentración de la pulpa de $2.5 \%$ ( $\mathrm{p} / \mathrm{p}$ ) y una concentración de colector de $3 \mathrm{ml} / \mathrm{kg}$ de carbón. Para incrementar el tiempo de permanencía de las partículas, se situó un flujo de recirculación a una distancia de 0.65 m medida desde el punto de alimentación. De acuerdo con el diseño experimental que se presenta en la Sección 2.4, se trabajó con 5 valores del flujo de aire en un rango entre 828 y $2419 \mathrm{ml} / \mathrm{min}$, en tanto que el flujo de alimentación se mantuvo constante en $2920 \mathrm{ml} / \mathrm{min}$. El flujo de fondo se ajustó según el flujo de aire, garantizando la altura deseada de $0.23 \mathrm{~m}$ sobre la alimentación de la pulpa, en tanto que el flujo de espumante se mantuvo constante en $42.19 \mathrm{ml} / \mathrm{min}$. 


\subsection{Diseño experimental}

En este trabajo se utilizó un diseño factorial $2^{3}$ central compuesto para evaluar el efecto de las condiciones usadas en la columna de flotación sobre las constantes cinéticas de cuatro muestras de carbón beneficiadas. En la Figura 2 se muestra el esquema del diseño experimental utilizado. Las variables independientes seleccionadas para el diseño experimental fueron el $\mathrm{pH}$ de la pulpa $(\mathrm{pH})$, la concentración de espumante $C_{E}$ y la velocidad del aire $J_{g}$ (con la cual se ajusta el flujo volumétrico del aire). En la Tabla 1 se muestran las condiciones de operación usadas, en tanto que en la Tabla 2 se muestran los valores y la nomenclatura asignada a las muestras de carbón. A cada carbón le fue asignado el código $Y_{i j k}$, donde $i$ indica la velocidad del aire (flujo volumétrico), $j$ indica el $\mathrm{pH}$ de la pulpa $\mathrm{y} k$ indica la concentración de espumante.

El pH se escogió como variable independiente por su influencia sobre las propiedades superficiales del carbón y por las interacciones entre las partículas de carbón y las burbujas, mientras que la concentración de espumante y la velocidad del aire se seleccionaron debido a la influencia que ejercen sobre la hidráulica de la columna y el diámetro de las burbujas. Además, el flujo de aire produce un ambiente estable para atrapar las partículas hidrofóbicas de carbón.
El error experimental está basado en experimentos repetidos en los puntos centrales del diseño experimental y fue de $\pm 5 \%$.

\section{Resultados y discusión}

\subsection{Caracterización de las muestras}

En la Tabla 3 se muestra el resultado de los análisis próximos de las muestras, realizados de acuerdo con las normas ASTM. Con los resultados encontrados y con la clasificación del rango de carbones ASTM, se encontró que los carbones Cerrejón y La Jagua son de tipo bituminoso alto en volátiles, mientras que los carbones Nechí y Guachinte son subituminoso B (Leonard, 1991).

Los resultados de la Tabla 3 muestran que el carbón Guachinte presenta el mayor contenido de ceniza (31.37\%) seguido por Cerrejón (18.53\%), La Jagua (3.94\%) y Nechí (7.66\%). En términos del contenido de azufre, el carbón Nechí mostró el menor valor $(0.4 \%)$, mientras que el carbón del Cerrejón presentó el mayor contenido de este (1.41\%). Los carbones La Jagua y Guachinte muestran valores relativamente cercanos de contenidos de azufre. Analizando los valores del poder calorífico encontrados, se observa que los carbones La Jagua y Nechí poseen los mayores valores de poder calorífico, lo cual se debe a sus bajos contenidos de cenizas; lo contrario ocurre con los carbones Cerrejón y Guachinte.

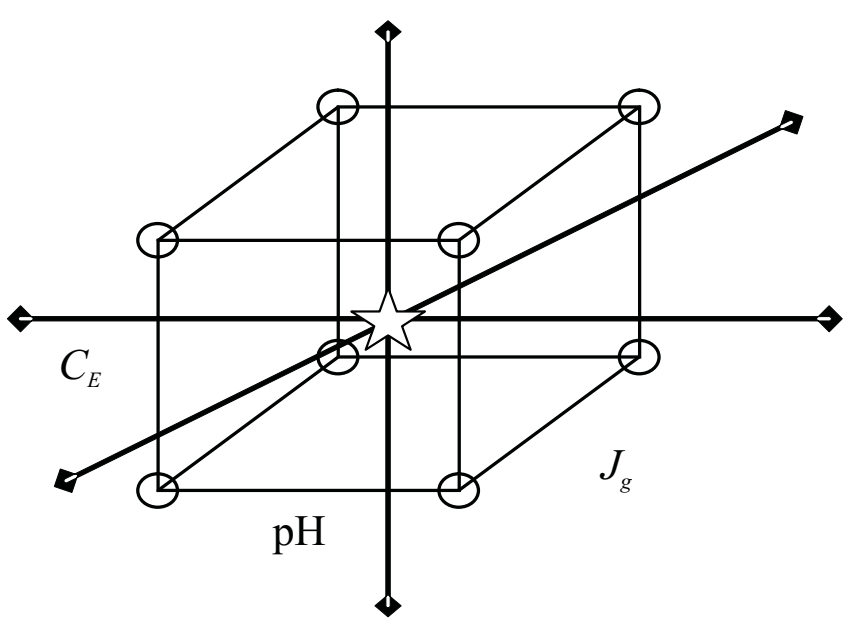

Figura 2. Esquema del diseño experimental utilizado. 
Tabla 1. Condiciones de operación de la columna de flotación.

\begin{tabular}{ll}
\hline \multicolumn{1}{c}{ Parámetros } & \multicolumn{1}{c}{ Valores } \\
\hline Flujo volumétrico de alimento & $2920 \mathrm{ml} / \mathrm{min}$ \\
Flujo volumétrico de aire & $828-2419 \mathrm{ml} / \mathrm{min}$ \\
Flujo volumétrico de agua de lavado & $160 \mathrm{ml} / \mathrm{min}$ \\
Flujo volumétrico de espumante & $42.19 \mathrm{ml} / \mathrm{min}$ \\
Dosificación de espumante & $2-6 \mathrm{ml} / \mathrm{kg}$ de carbón \\
$\mathrm{pH}$ & $4-10$ \\
\hline
\end{tabular}

Tabla 2. Significado de la nomenclatura utilizada.

\begin{tabular}{ccl}
\hline Subindice & Nivel & \multicolumn{1}{c}{ Significado } \\
\hline$i$ & 0 & Punto medio $(1.4 \mathrm{~cm} / \mathrm{s})$ \\
& 1 & Nivel bajo $(1.0 \mathrm{~cm} / \mathrm{s})$ \\
2 & Nivel alto $(1.8 \mathrm{~cm} / \mathrm{s})$ \\
& 3 & Punto axial inferior $(0.7 \mathrm{~cm} / \mathrm{s})$ \\
& 4 & Punto axial superior $(2.1 \mathrm{~cm} / \mathrm{s})$ \\
\hline$j$ & 0 & Punto medio $(7)$ \\
& 1 & Nivel bajo (5) \\
& 2 & Nivel alto $(9)$ \\
& 3 & Punto axial inferior $(4)$ \\
& 4 & Punto axial superior $(10)$ \\
\hline$k$ & 0 & Punto medio $(4 \mathrm{ml} / \mathrm{kg})$ \\
& 1 & Nivel bajo $(3 \mathrm{ml} / \mathrm{kg})$ \\
& 2 & Nivel alto $(5 \mathrm{ml} / \mathrm{kg})$ \\
& 3 & Punto axial inferior $(2 \mathrm{ml} / \mathrm{kg})$ \\
& 4 & Punto axial superior $(6 \mathrm{ml} / \mathrm{kg})$ \\
\hline
\end{tabular}

Tabla 3. Análisis próximos de las muestras originales.

\begin{tabular}{lccrc}
\hline Análisis $^{\text {a }}$ & Cerrejón & La Jagua & Nechí & Guachinte \\
\hline Materia volátil (\%) & 29.17 & 38.99 & 45.14 & 29.81 \\
Cenizas (\%) & 18.53 & 3.94 & 7.66 & 31.37 \\
Azufre (\%) & 1.41 & 1.00 & 0.40 & 0.84 \\
Poder calorífico (Btu / lb) & 10210 & 13871 & 11629 & 9103 \\
\hline
\end{tabular}

a: En base seca

\subsection{Determinación de la constante cinética}

En la determinación de la constante cinética de flotación, se utilizó la Ec. (2) bajo condiciones de flujo pistón en la columna. Este régimen lo determina el número de dispersión y se ha reportado (Levenspiel, 1972) que con valores de este número tendientes a cero, las condiciones se encuentran dentro de este régimen. En la Tabla 4 se muestran los valores del número de dispersión $N_{d}$ y del número de Reynolds $R e_{b}$ para los 4 carbones usando diferentes relaciones de velocidad del aire a concentración de espumante $J_{g} / C_{E}$. En general, para todas las condiciones de operación, $N_{d}$ tiende a cero, lo cual indica que la columna de flotación trabajó en régimen de flujo pistón. 
Tabla 4. Parámetros hidráulicos.

\begin{tabular}{cccc}
\hline$J_{g} / C_{E}$ & $D_{b}(\mathrm{~mm})$ & $R e_{b}$ & $N_{d}$ \\
\hline $1.0 / 3$ & 1.03 & 56 & 0.028 \\
$1.0 / 5$ & 1.03 & 56 & 0.027 \\
$1.0 / 3$ & 1.03 & 56 & 0.028 \\
$1.0 / 5$ & 1.03 & 56 & 0.027 \\
$1.8 / 3$ & 1.39 & 100 & 0.032 \\
$1.8 / 5$ & 1.39 & 100 & 0.031 \\
$1.8 / 3$ & 1.39 & 100 & 0.032 \\
$1.8 / 5$ & 1.39 & 100 & 0.031 \\
$1.4 / 4$ & 1.27 & 83 & 0.030 \\
$1.4 / 4$ & 1.27 & 83 & 0.030 \\
$1.4 / 4$ & 1.27 & 83 & 0.030 \\
$1.4 / 4$ & 1.27 & 83 & 0.030 \\
$1.4 / 4$ & 1.27 & 83 & 0.030 \\
$0.7 / 4$ & 0.85 & 39 & 0.025 \\
$2.1 / 4$ & 1.51 & 117 & 0.032 \\
$1.4 / 4$ & 1.27 & 83 & 0.030 \\
$1.4 / 4$ & 1.27 & 83 & 0.030 \\
$1.4 / 2$ & 1.28 & 85 & 0.030 \\
$1.4 / 6$ & 1.27 & 84 & 0.029 \\
\hline
\end{tabular}

$J_{g}$ (velocidad del aire, $\mathrm{cm} / \mathrm{s}$ ), $C_{E}$ (concentración de espumante, $\mathrm{ml} / \mathrm{kg}$ de carbón), $D_{b}$ (diámetro de la burbuja, $\mathrm{mm}$ ), $\mathrm{Re}_{\mathrm{b}}$ (número de Reynolds), $\mathrm{N}_{\mathrm{d}}$ (número de dispersión). $D_{b}$ fue calculado utilizando la ecuación de Yianatos et al. (1988).

Tabla 5. Significancia estadística de las variables y sus interacciones.

\begin{tabular}{|c|c|c|c|c|c|c|}
\hline Fuente & gl & $\begin{array}{r}F \text { calculado } \\
\text { (Cerrejón) }\end{array}$ & $\begin{array}{c}F \text { calculado } \\
\text { (Nechí) }\end{array}$ & $\begin{array}{r}F \text { calculado } \\
\text { (La Jagua) }\end{array}$ & $\begin{array}{l}F \text { calculado } \\
\text { (Guachinte) }\end{array}$ & $F$ crítico \\
\hline Modelo & 13 & 13.339 & 29.379 & 21.956 & 11.082 & 2.3 \\
\hline A & 1 & 16.174 & 2.421 & 0.350 & 0.297 & 6.6 \\
\hline B & 1 & 0.798 & 56.005 & 78.947 & 48.359 & 6.6 \\
\hline $\mathrm{C}$ & 1 & 0.004 & 5.702 & 27.810 & 6.038 & 6.6 \\
\hline $\mathrm{AB}$ & 1 & 1.100 & 0.018 & 0.804 & 0.164 & 6.6 \\
\hline $\mathrm{AC}$ & 1 & 4.717 & 0.392 & 5.983 & 0.064 & 6.6 \\
\hline $\mathrm{BC}$ & 1 & 0.035 & 0.052 & 6.071 & 24.240 & 6.6 \\
\hline $\mathrm{A}^{2}$ & 1 & 5.461 & 1.028 & 0.294 & 0.078 & 6.6 \\
\hline $\mathrm{B}^{2}$ & 1 & 48.514 & 127.183 & 7.215 & 11.206 & 6.6 \\
\hline $\mathrm{C}^{2}$ & 1 & 0.133 & 15.170 & 1.128 & 3.614 & 6.6 \\
\hline $\mathrm{ABC}$ & 1 & 0.331 & 8.308 & 6.592 & 2.770 & 6.6 \\
\hline$A^{3}$ & 1 & 0.898 & 2.933 & 2.167 & 0.456 & 6.6 \\
\hline $\mathrm{B}^{3}$ & 1 & 5.914 & 14.874 & 7.285 & 11.857 & 6.6 \\
\hline $\mathrm{C}^{3}$ & 1 & 1.547 & 10.393 & 19.078 & 10.942 & 6.6 \\
\hline Residual & 5 & & & & & \\
\hline Ajuste & 1 & 2.187 & 0.128 & 0.385 & 0.036 & 7.7 \\
\hline Error & 4 & & & & & \\
\hline Total & 18 & & & & & \\
\hline $\begin{array}{l}\mathrm{gl}=\text { grado } \\
\mathrm{A}=\text { Veloci } \\
\mathrm{B}=\text { pH de } \\
\mathrm{C}=\text { Conce }\end{array}$ & lp & snumant & & & & \\
\hline
\end{tabular}




\subsection{Análisis estadístico}

Los experimentos en la columna de flotación se realizaron de acuerdo con el diseño experimental. Un total de 19 corridas experimentales se desarrollaron para cada muestra de carbón. Los resultados de la significancia de las variables y sus interacciones se presentan en la Tabla 5. La significancia de los principales efectos y sus interacciones se evalúan de acuerdo con el procedimiento descrito por Montgomery (1999) y Hicks (1982). Los datos se analizaron estadísticamente utilizando el programa Design Expert. Se definió la siguiente notación: $\mathrm{A}=$ velocidad del aire, $\mathrm{B}=\mathrm{pH}$ de la pulpa, $\mathrm{C}=$ concentración de espumante, $\mathrm{Y}=$ constante cinética. Las superficies de respuestas obtenidas son las siguientes:

Cerrejón $\left(\right.$ con $\left.\mathrm{R}^{2}=0.97\right)$

$$
\begin{aligned}
1 / Y & =3.71+0.83 A+0.18 B-0.013 C \\
& -0.22 A^{2}+0.65 B^{2}+0.034 C^{2} \\
& +0.13 A B+0.27 A C-0.023 B C \\
& -0.099 A^{3}+0.25 B^{3}+0.13 C^{3} \\
& -0.07 A B C
\end{aligned}
$$

Nechí $\left(\operatorname{con} \mathrm{R}^{2}=0.99\right)$

$$
\begin{aligned}
1 / Y & =3.58+0.19 A-0.92 B+0.3 C \\
& +0.057 A^{2}+0.64 B^{2}+0.22 C^{2} \\
& -9.927 \times 10^{-3} A B+0.046 A C-0.017 B C \\
& -0.11 A^{3}+0.24 B^{3}+0.20 C^{3}-0.21 A B C
\end{aligned}
$$

La Jagua $\left(\operatorname{con} \mathrm{R}^{2}=0.98\right)$

$$
\begin{aligned}
1 / Y & =4.38+0.10 A+1.54 B+0.91 C \\
& +0.043 A^{2}-0.21 B^{2}-0.084 C^{2} \\
& -0.092 A B-0.25 A C+0.25 B C \\
& +0.13 A^{3}-0.24 B^{3}+0.38 C^{3}-0.26 A B C
\end{aligned}
$$

Guachinte $\left(\operatorname{con} \mathrm{R}^{2}=0.97\right)$

$$
\begin{aligned}
1 / Y & =3.55+0.095 A-1.21 B-0.43 C \\
& +0.022 A^{2}+0.27 B^{2}+0.15 C^{2} \\
& +0.042 A B+0.026 A C+0.51 B C \\
& +0.06 A^{3}+0.3 B^{3}+0.29 C^{3} \\
& +0.17 A B C
\end{aligned}
$$

En las Ecs. (4) a (7), $\mathrm{R}^{2}$ es el coeficiente de correlación. Los datos de la Tabla 5 muestran que para el modelo se tiene un efecto significativo $(F>F$ crítico), mientras que para la carencia de ajuste se muestra un efecto no significativo $(F<F$ crítico), indicando que la expresión encontrada es una aproximación razonable para la superficie en la región considerada en el experimento (Montgomery, 1999; Hicks, 1982).

En la Tabla 5 se observa que el pH tiene un efecto significativo en los carbones La Jagua, Nechí y Guachinte, mientras que el flujo de aire tiene significancía en el carbón Cerrejón, en tanto que la concentración de espumante mostró un efecto significativo sobre el carbón La Jagua, como se deduce de los valores de $F$ y $F$ crítico (Montgomery, 1999; Hicks, 1982).

De la misma manera, se encontraron efectos de curvatura para las diferentes variables utilizadas, mostrando que el $\mathrm{pH}$ tiene efecto significativo para los términos cuadráticos en las cuatro muestras de carbón. Por otro lado, el término cuadrático de la concentración de espumante mostró significancía para el carbón Nechí. Con respecto a los efectos cúbicos se tienen efectos significativos para las muestras Nechí, La Jagua y Guachinte para el pH y la concentración de espumante. En cuanto a los efectos cruzados, se encontró significancía en el carbón Guachinte para las variables $\mathrm{pH}$ y concentración de espumante y en el carbón Nechí para las tres variables. 


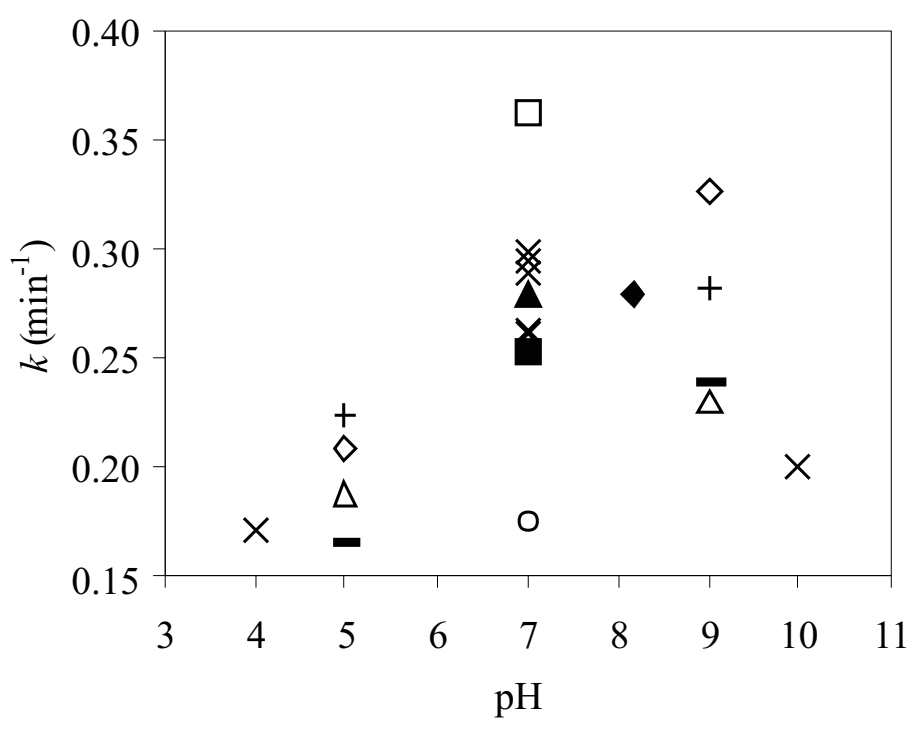

- $J_{g} 0.7 \mathrm{~cm} / \mathrm{s}, C_{E} 4 \mathrm{ml} / \mathrm{kg} ; \diamond J_{g} 1.0 \mathrm{~cm} / \mathrm{s}, C_{E} 3 \mathrm{ml} / \mathrm{kg} ; \Delta J_{g} 1.0 \mathrm{~cm} / \mathrm{s}, C_{E} 5 \mathrm{ml} / \mathrm{kg} ;$ $\mathrm{X} J_{g} 1.4 \mathrm{~cm} / \mathrm{s}, C_{E} 4 \mathrm{ml} / \mathrm{kg} ; \square J_{g} 1.4 \mathrm{~cm} / \mathrm{s}, C_{E} 2 \mathrm{ml} / \mathrm{kg} ; O J_{g} 1.4 \mathrm{~cm} / \mathrm{s}, C_{E} 6 \mathrm{ml} / \mathrm{kg} ;$ $+J_{g} 1.8 \mathrm{~cm} / \mathrm{s}, C_{E} 3 \mathrm{ml} / \mathrm{kg} ;-J_{g} 1.8 \mathrm{~cm} / \mathrm{s}, C_{E} 5 \mathrm{ml} / \mathrm{kg} ; \Delta J_{g} 2.1 \mathrm{~cm} / \mathrm{s}, C_{E} 4 \mathrm{ml} / \mathrm{kg}$; - $J_{\mathrm{g}} 1.0 \mathrm{~cm} / \mathrm{s}, C_{E} 3 \mathrm{ml} / \mathrm{kg}$.

Figura 3. Constante cinética vs. pH para el carbón Nechí.

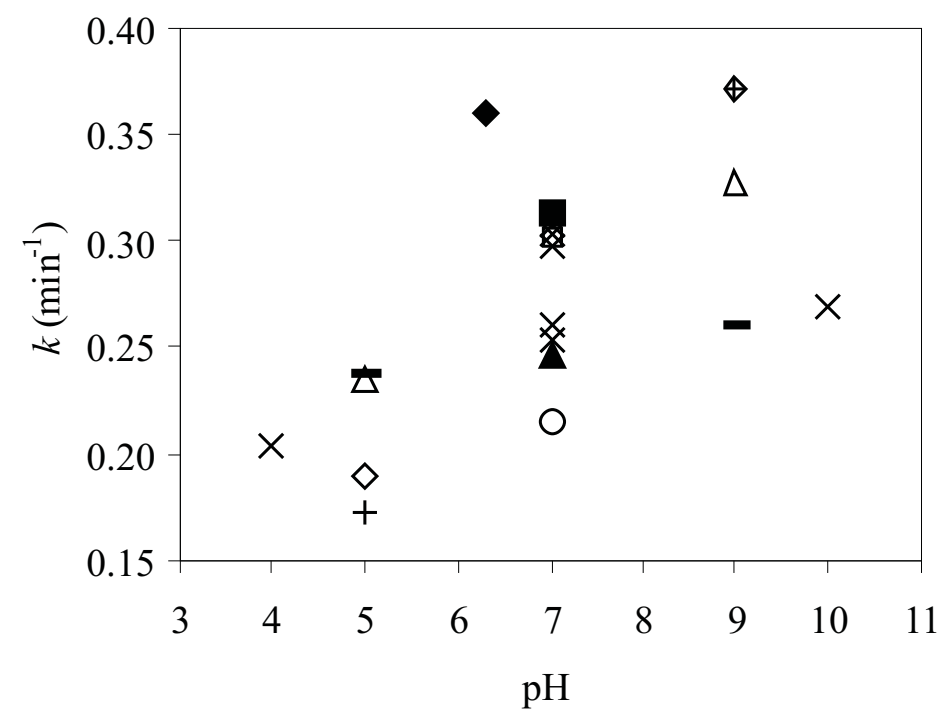

- $J_{g} 0.7 \mathrm{~cm} / \mathrm{s}, C_{E} 4 \mathrm{ml} / \mathrm{kg} ; \diamond J_{g} 1.0 \mathrm{~cm} / \mathrm{s}, C_{E} 3 \mathrm{ml} / \mathrm{kg} ; \Delta J_{g} 1.0 \mathrm{~cm} / \mathrm{s}, C_{E} 5 \mathrm{ml} / \mathrm{kg} ;$ $\mathrm{X} J_{g} 1.4 \mathrm{~cm} / \mathrm{s}, C_{E} 4 \mathrm{ml} / \mathrm{kg} ; \square J_{g} 1.4 \mathrm{~cm} / \mathrm{s}, C_{E} 2 \mathrm{ml} / \mathrm{kg} ; O J_{g} 1.4 \mathrm{~cm} / \mathrm{s}, C_{E} 6 \mathrm{ml} / \mathrm{kg} ;$ $+J_{g} 1.8 \mathrm{~cm} / \mathrm{s}, C_{E} 3 \mathrm{ml} / \mathrm{kg} ;-J_{g} 1.8 \mathrm{~cm} / \mathrm{s}, C_{E} 5 \mathrm{ml} / \mathrm{kg} ; \Delta J_{g} 2.1 \mathrm{~cm} / \mathrm{s}, C_{E} 4 \mathrm{ml} / \mathrm{kg} ;$ $\rightarrow J_{g} 1.0 \mathrm{~cm} / \mathrm{s}, C_{E} 3 \mathrm{ml} / \mathrm{kg}$.

Figura 4. Constante cinética vs. pH para el carbón Guachinte. 


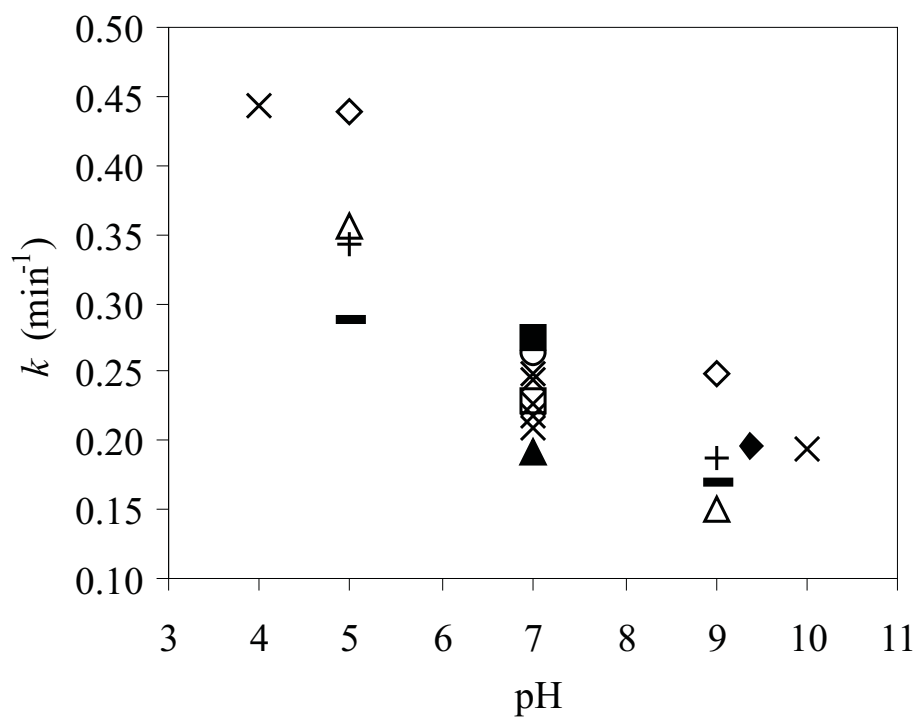

- $J_{g} 0.7 \mathrm{~cm} / \mathrm{s}, C_{E} 4 \mathrm{ml} / \mathrm{kg} ; \Delta J_{g} 1.0 \mathrm{~cm} / \mathrm{s}, C_{E} 3 \mathrm{ml} / \mathrm{kg} ; \Delta J_{g} 1.0 \mathrm{~cm} / \mathrm{s}, C_{E} 5 \mathrm{ml} / \mathrm{kg} ;$ $\mathrm{X} J_{\mathrm{g}} 1.4 \mathrm{~cm} / \mathrm{s}, C_{E} 4 \mathrm{ml} / \mathrm{kg} ; \square J_{g} 1.4 \mathrm{~cm} / \mathrm{s}, C_{E} 2 \mathrm{ml} / \mathrm{kg} ; O J_{g} 1.4 \mathrm{~cm} / \mathrm{s}, C_{E} 6 \mathrm{ml} / \mathrm{kg}$; $+J_{g} 1.8 \mathrm{~cm} / \mathrm{s}, C_{E} 3 \mathrm{ml} / \mathrm{kg} ;-J_{g} 1.8 \mathrm{~cm} / \mathrm{s}, C_{E} 5 \mathrm{ml} / \mathrm{kg} ; \Delta J_{g} 2.1 \mathrm{~cm} / \mathrm{s}, C_{E} 4 \mathrm{ml} / \mathrm{kg} ;$ $\checkmark J_{g} 1.0 \mathrm{~cm} / \mathrm{s}, C_{E} 3 \mathrm{ml} / \mathrm{kg}$.

Figura 5. Constante cinética vs. pH para el carbón La Jagua.

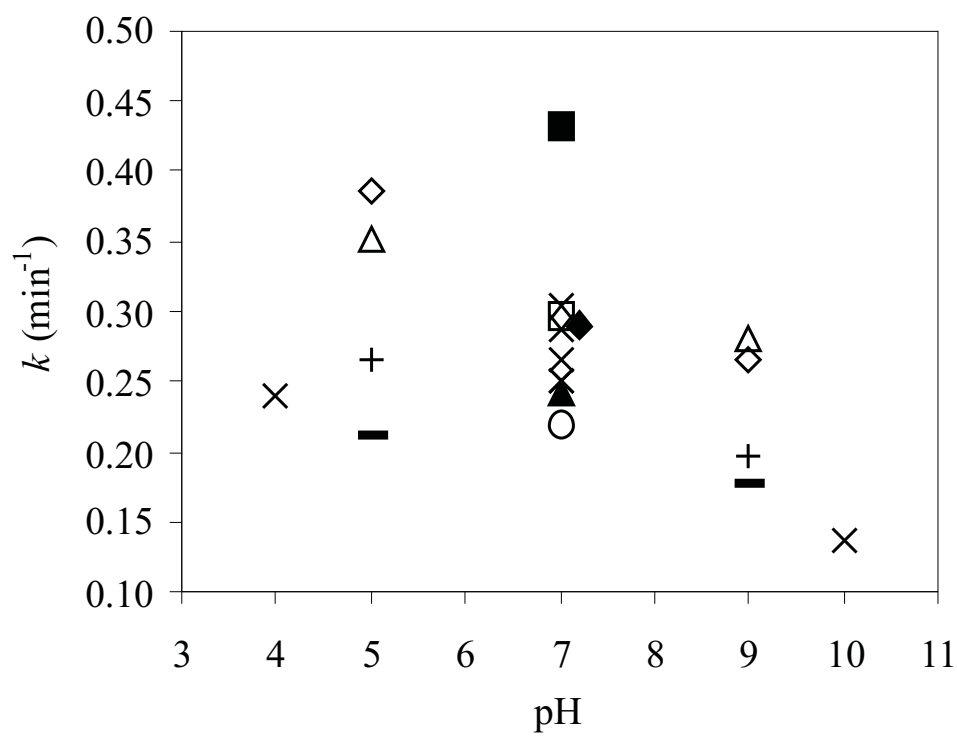

- $J_{g} 0.7 \mathrm{~cm} / \mathrm{s}, C_{E} 4 \mathrm{ml} / \mathrm{kg} ; \diamond J_{g} 1.0 \mathrm{~cm} / \mathrm{s}, C_{E} 3 \mathrm{ml} / \mathrm{kg} ; \Delta J_{g} 1.0 \mathrm{~cm} / \mathrm{s}, C_{E} 5 \mathrm{ml} / \mathrm{kg} ;$ $\mathrm{X} J_{g} 1.4 \mathrm{~cm} / \mathrm{s}, C_{E} 4 \mathrm{ml} / \mathrm{kg} ; \square J_{g} 1.4 \mathrm{~cm} / \mathrm{s}, C_{E} 2 \mathrm{ml} / \mathrm{kg} ; O J_{g} 1.4 \mathrm{~cm} / \mathrm{s}, C_{E} 6 \mathrm{ml} / \mathrm{kg} ;$ $+J_{g} 1.8 \mathrm{~cm} / \mathrm{s}, C_{E} 3 \mathrm{ml} / \mathrm{kg} ;-J_{g} 1.8 \mathrm{~cm} / \mathrm{s}, C_{E} 5 \mathrm{ml} / \mathrm{kg} ; \Delta J_{g} 2.1 \mathrm{~cm} / \mathrm{s}, C_{E} 4 \mathrm{ml} / \mathrm{kg} ;$ $\checkmark J_{g} 1.0 \mathrm{~cm} / \mathrm{s}, C_{E} 3 \mathrm{ml} / \mathrm{kg}$.

Figura 6. Constante cinética vs. pH para el carbón Cerrejón. 


\subsection{Efecto del $\mathrm{pH}$, velocidad del aire $\mathrm{y}$ concentración de espumante sobre la constante cinética de flotación}

Las Figuras 3 a 6 muestran las constantes cinéticas de las cuatro muestras de carbón calculadas a partir de la Ec. (2) para las condiciones de operación dadas en la Tabla 1.

Se observa que en la mayoría de las condiciones de operación, las constantes cinéticas de los carbones Nechí y Guachinte tienden a aumentar con el incremento del $\mathrm{pH}$, mientras que, en los carbones La Jagua y Cerrejón se tiene un efecto contrario. Las constantes cinéticas a $\mathrm{pH} 9$ mostraron altos valores para los carbones Nechí $\left(0.33 \mathrm{~min}^{-1}\right)$ y para el carbón Guachinte $\left(0.37 \mathrm{~min}^{-1}\right)$. No obstante, el carbón Nechí presentó el mayor valor $\left(0.36 \mathrm{~min}^{-1}\right)$ a $\mathrm{pH}$ 7. Los carbones Cerrejón y La Jagua presentaron altos valores de la constante cinética en medio ácido $\left(0.39 \mathrm{~min}^{-1}\right.$ a pH 5 y $0.44 \mathrm{~min}^{-1}$ a $\mathrm{pH} 4$, respectivamente). Sin embargo, el mayor valor para el carbón Cerrejón fue $0.43 \mathrm{~min}^{-1}$ a $\mathrm{pH}$ 7. Se destaca el hecho de que al procesar muestras de carbón sin adición de reactivos $(\mathrm{NaOH}, \mathrm{HCl})$, se obtuvieron valores aceptables de la constante cinética (alrededor de $0.3 \mathrm{~min}^{-1}$ ) para los carbones Cerrejón, Nechí y Guachinte. Estos resultados son importantes desde el punto de vista económico del proceso.

La disminución en la constante cinética con el incremento del $\mathrm{pH}$ mostrada para los carbones Cerrejón y La Jagua sugiere la presencia de grupos carboxilos $(\mathrm{COOH})$, carbonilos $(\mathrm{C}=\mathrm{O})$ y fenólicos polares en esos carbones, grupos que incrementan la hidratación a través de los enlaces de hidrógeno con las moléculas de agua, así como la precipitación en la superficie del carbón de especies minerales tales como $\mathrm{Fe}(\mathrm{OH})_{2}$ y $\mathrm{Fe}(\mathrm{OH})_{3}$. Para los carbones Guachinte y Nechí, ocurre el efecto contrario, es decir, con el incremento del $\mathrm{pH}$ no hay ionización de los grupos carboxílicos, carbonilos y fenólicos polares en su superficie, posiblemente debido a la naturaleza compleja de estos grupos con la superficie del carbón o a que pueden estar unidos con alguna estructura química compleja, disminuyendo la hidratación, e indicando que las especies minerales se disuelven en la superficie del carbón
(Tao et al., 2002; Liu et al., 1994a; Liu et al., 1994b).

Por otro lado, el número de grupos funcionales que contienen oxígeno varía según el grado de carbonización. Como puede verse de los análisis próximos de los carbones, las muestras presentan diferentes grados de carbonización. Es de notar que el $\mathrm{pH}$ es un factor importante en la flotación del carbón, donde los iones $\mathrm{OH}^{-}$y $\mathrm{H}^{+}$se sabe que afectan el potencial de superficie, actuando sobre la superficie del carbón al cambiar el grado de polaridad y la estructura de la doble capa entre el agua y el carbón (Tao et al., 2002; Liu et al., 1994a; Liu et al., 1994b; Honaker et al., 1996).

Las Figuras 3 a 6 muestran que, en general, para las cuatro muestras de carbón, al incrementarse la concentración de espumante y la velocidad del aire, existe la tendencia de que la constante cinética disminuya. Los máximos valores se encontraron cuando se adicionaron de 2 a $4 \mathrm{ml} / \mathrm{kg}$ de carbón de espumante y velocidad de aire entre 0.7 y $1.4 \mathrm{~cm} / \mathrm{s}$. La adición de espumante podría favorecer la interfase aire / agua, actuando sobre la superficie de carbón a través de las interacciones hidrofóbicas entre la molécula del lauril éter sulfato de sodio y la superficie del carbón, por medio de los enlaces con el ión sodio de la molécula de espumante y los grupos funcionales en la superficie del carbón (Fuerstenau \& Pradip, 1982).

Lo anterior sugiere que existe un ambiente favorable para la adhesión partícula de carbónburbuja de aire, generando de esta manera una espuma estable. Finch \& Dobby (1990) mostraron que el incremento en la concentración del espumante genera tamaños pequeños de burbuja favoreciendo los procesos de colisión y adhesión (partícula-burbuja), afectando de manera directa la constante cinética.

A partir de los datos de concentración de espumante y velocidad del aire se calculó el diámetro de las burbujas, obteniéndose un rango de diámetro de burbuja entre 0.85 y $1.51 \mathrm{~mm}$. Tal como se presenta en las Figuras 3 a 6 , pequeños valores de velocidad del aire y concentración de espumante generan un incremento en la constante cinética. Para los cuatro carbones, altos valores de 
la constante cinética fueron obtenidos con un rango de la velocidad del aire entre 0.7 y $1.4 \mathrm{~cm} / \mathrm{s}$ con concentración de espumante de 3 y $4 \mathrm{ml} / \mathrm{kg}$.

El aumento de la constante cinética al disminuir la velocidad del aire y la concentración de espumante (o sea, al disminuir el diámetro de la burbuja), es consistente con la Ec. (3), que indica que la constante cinética varía con el recíproco del diámetro de la burbuja al cubo ( $k$ varia con $1 / D_{b}{ }^{3}$ ), como se puede observar en la Tabla 4.

El efecto de las variables utilizadas en el proceso de flotación sobre la constante cinética, indica que la flotabilidad de los cuatros carbones se afecta de manera directa por la velocidad del aire, $\mathrm{pH}$ de la pulpa y concentración de espumante, como puede deducirse de los resultados presentados en la Tabla 5. Las diferencias en los efectos de estas variables sobre cada carbón se deben a las propiedades superficiales, hidrodinámica de la columna de flotación, hidrofobicidad y al contenido de grupos funcionales con oxígeno de cada carbón, así como también a los diferentes grados de carbonización y distribución de grupos macerales que estos presentan (como consecuencia de las diferentes características y condiciones geológicas, geográficas, climatológicas y biológicas durante su formación).

\section{Conclusiones}

En general, las condiciones de $\mathrm{pH}$ neutro, valores bajos de velocidad del aire y concentración de espumante son favorables para obtener altos valores de la constante cinética.

Altos valores de la constante cinética se obtuvieron para diámetros de burbuja entre $0.85 \mathrm{~mm}$ y $1.3 \mathrm{~mm}$, mostrando que para bajos flujos de aire se obtienen pequeños diámetros de burbujas, incrementándose la recuperación de material combustible debido al incremento en el área de superficie, aumentando, por lo tanto, la probabilidad de contacto entre partículas y burbujas.
Las diferencias en los valores de las constantes cinéticas se deben principalmente a las diferencias en la flotabilidad de cada carbón como consecuencia de los distintos grados de carbonización que presentan las muestras (Tabla 3) así como posiblemente también, a la composición petrográfica, grupos funcionales, distribución mineralógica, modo y tipo de ocurrencia de estos minerales.

\section{Agradecimientos}

Los autores agradecen al Instituto Colombiano de Ciencia y Tecnología (Colciencias) por el apoyo económico al estudiante Jorge Luís Piñeres a través de la convocatoria para Doctorados Nacionales 2005 y al proyecto Separación de macerales de carbones colombianos usando flotación en columna, con código 1106-06-17318.

\section{Referencias bibliograficas}

Blaschke, W. S. (1996). New trends in coal preparation technologies and equipment. Gordon and Breach Publishers.

Dobby, G. S., \& Finch, J. A. (1986). Flotation column scale-up and modelling. Canadian Institute of Mining, Metallurgy and Petroleum (CIM) Bulletin 79 (889), 89-96.

Finch, J.A., \& Dobby, G. S. (1990) Column flotation. Elsevier Science Pub.

Fuerstenau, D. W., \& Pradip. (1982). Adsorption of frothers at coal/water interfaces. Colloids and Surfaces 4 (3), 213-227.

Hicks, C. R. (1982). Fundamental concepts in the design of experiments. Saunders College Publishing.

Honaker, R. Q., Mohanty, M. K., \& Crelling, J. C. (1996). Coal maceral separation using column flotation. Minerals Engineering 9 (4), 449-464.

Leonard, J. W. (editor) (1991). Coal preparation. Society for Mining, Metallurgy, and Exploration, Inc. 
Levenspiel, O. (1972). Chemical reaction engineering, John Wiley \& Sons.

Liu, D., Somasundaran, D., Vasudevan, T. V., \& Harris, C. C. (1994a). Role of $\mathrm{pH}$ and dissolved mineral species in Pittsburg $\mathrm{N}^{\circ} 8$ coal flotation system. I Floatability of coal. International Journal of Mineral Processing 41 (3-4), 201-214.

Liu, D., Somasundaran, D., Vasudevan, T. V., \& Harris, C. C. (1994b). Role of $\mathrm{pH}$ and dissolved mineral species in Pittsburg $\mathrm{N}^{\circ} 8$ coal flotation system. II Separation of pyrite and non-pyritic minerals from coal. International Journal of Mineral Processing 41 (3-4), 215-225.

Montgomery, D. C. (1999). Diseño y análisis de experimentos. Grupo Editorial Iberoamérica.

Tao, D., Li, B., Johnson, S., \& Parehk, B. (2002). A flotation study of refuse pond coal slurry. Fuel Processing Technology 76 (3), 201-210.

Yianatos, J. B., Finch, J. A., Dobby, G. S., \& Manqui, X. (1988). Bubble size estimation in bubble swarm. Journal of Colloid and Interface Science 126(1), 37-44.

Yoon, R.-H., \& Mao, L. (1996). Application of extended DLVO theory. IV: Derivation of flotation rate equation from first principles. Journal of Colloid and Interface Science 181 (2), 613-626. 